\title{
Analytical Green's function of the multidimensional frequency-dependent phonon Boltzmann equation
}

\author{
Chengyun Hua and Austin J. Minnich* \\ Division of Engineering and Applied Science, California Institute of Technology, Pasadena, California 91125, USA
}

(Received 19 August 2014; revised manuscript received 20 November 2014; published 31 December 2014)

\begin{abstract}
Thermal phonon transport at length scales comparable to mean free paths is governed by the Boltzmann equation, which is challenging to solve due to its high dimensionality. Here, we present an analytical Green's function for the frequency-dependent, multidimensional Boltzmann equation under the relaxation-time approximation. The new analytical solution is valid from diffusive to ballistic transport regimes and rigorously includes frequency dependence of phonon properties. We demonstrate that our result enables simple closed-form solutions for a number of multidimensional problems for which the only prior solution methods have been computationally expensive numerical simulations.
\end{abstract}

DOI: 10.1103/PhysRevB.90.214306

PACS number(s): 65.40.-b

\section{INTRODUCTION}

Heat conduction at length scales comparable to phonon mean free paths (MFPs) has been of considerable interest recently [1] due to its applications in many technologies such as thermoelectrics [2,3] and electronic devices [4]. At these small scales, classic continuum transport theories such as Fourier's law are not valid due to the absence of scattering and therefore a local temperature [5]. In this nondiffusive regime, phonon transport is nonlocal and is described by the Boltzmann transport equation (BTE) [6].

The BTE is an integrodifferential equation of time, real space, and phase space, and it describes a wide range of phenomena in many fields ranging from astronomy to neutron transport [7-10]. The equation was first used to describe phonons in solids by Peierls in 1929 [11]. In 1958, Englman obtained analytical solutions of the BTE with simpler collision terms to describe the transport theory of temperature waves in insulators [12]. Later, Williams extended Englman's theory and obtained simpler forms of the solution [13]. In the 1960s and 1970s, Simons developed the theory of high-frequency thermal waves in dielectrics in which phonons can undergo both normal and resistive interaction processes based on the BTE [14]. A decade later, the equation was reinvestigated by Mahan and Claro [15] to study the nonlocal heat transfer effects during transient heating. Majumdar derived an equation of phonon radiative transfer from the BTE [5], which was applied to study heat transfer across diamond thin films for both steady-state and transient cases [16]. Chen demonstrated the failure of Fourier's law in nonplanar nanostructures and provided a general solution for the temperature rise of heatgenerating nanometer and micrometer particles embedded in a host medium based on the BTE [17]. Later, he derived ballistic-diffusive heat conduction equations from the BTE which gave a better approximation than the Fourier law at small scales but was much simpler to solve than the BTE [18]. This two-channel model was then employed to solve several planar nanoscale heat conduction problems [19]. Recently, Miranda et al. obtained a constitutive equation for heat conduction

\footnotetext{
*aminnich@caltech.edu
}

derived from the exact solution of the BTE by a series expansion [20].

Despite these extensive efforts to study phonon transport using the BTE, most of the previous approaches depended on simplifications to make the problem tractable. For example, all the works mentioned above assumed a single-phonon mean free path even though recent work has demonstrated that the transport properties of phonons in solids vary widely over the broad thermal spectrum $[21,22]$. Further, the above solutions assumed simple one-dimensional (1D) geometries that do not often occur in practice. A number of numerical approaches can address these issues [5,19,23-26], particularly variancereduced Monte Carlo methods recently introduced by Peraud and Hadjiconstantinou [27,28]. However, analytical solutions to the BTE including these additional effects have not been reported.

In this work, we demonstrate that exact analytical solutions to the multidimensional, frequency-dependent transient BTE for arbitrary heat inputs can be obtained using Fourier transforms in infinite or semi-infinite domains. Our result provides simple, closed-form solutions to multidimensional problems that have previously been possible to solve only with computationally expensive numerical simulations or inaccurate simplifications. Solutions to specific problems can be obtained more than three orders of magnitude faster than prior numerical approaches based on discrete ordinates. Moreover, our identification of an analytical solution enables physical insight to be obtained just by examining the equations algebraically and thus represents a useful advance toward understanding nondiffusive heat conduction.

\section{DERIVATION}

The frequency-dependent BTE under relaxation-time approximation is given by

$$
\frac{\partial g_{\omega}}{\partial t}+\mathbf{v}_{g} \cdot \nabla g_{\omega}=-\frac{g_{\omega}-g_{0}(T)}{\tau_{\omega}}+\frac{Q_{\omega}}{4 \pi},
$$

where $g_{\omega}=\hbar \omega D(\omega)\left[f_{\omega}(\mathbf{r}, t, \theta, \phi)-f_{0}\left(T_{0}\right)\right]$ is the desired deviational distribution function, $g_{0}(T)$ is the equilibrium deviational distribution function defined below, $Q_{\omega}(\mathbf{r}, t)$ is the spectral volumetric heat generation, $\mathbf{v}_{g}(\omega)$ is the phonon group velocity, and $\tau_{\omega}$ is the phonon relaxation time. Here, $\mathbf{r}$ is the 
spatial vector, $t$ is the time, $\omega$ is the phonon frequency, and $T(\mathbf{r}, t)$ is the local temperature. In the Cartesian coordinate system and assuming an isotropic crystal, the advection term in Eq. (1) is expanded as

$$
\begin{aligned}
\mathbf{v}_{g} \cdot \nabla g_{\omega}= & v_{g} \mu \frac{\partial g_{\omega}}{\partial z}+v_{g} \sqrt{1-\mu^{2}} \cos \phi \frac{\partial g_{\omega}}{\partial x} \\
& +v_{g} \sqrt{1-\mu^{2}} \sin \phi \frac{\partial g_{\omega}}{\partial y},
\end{aligned}
$$

where $\mu=\cos (\theta)$ is the directional cosine of the polar angle $\theta$ and $\phi$ is the azimuthal angle. Here, we emphasize that while many crystals contain minor anisotropies in the Brillouin zone the vast majority of crystals are thermally isotropic, with notable exceptions being materials with very anisotropic bonding such as graphite. We present a validation of this assertion for the $a b$ initio calculations for silicon used here in the Appendix.

Assuming a small temperature rise, $\Delta T(\mathbf{r}, t)=T(\mathbf{r}, t)-$ $T_{0}$, relative to a reference temperature $T_{0}$, the equilibrium deviational distribution is proportional to $\Delta T(\mathbf{r}, t)$ :

$g_{0}(T)=\frac{1}{4 \pi} \hbar \omega D(\omega)\left[f_{\mathrm{BE}}(T)-f_{\mathrm{BE}}\left(T_{0}\right)\right] \approx \frac{1}{4 \pi} C_{\omega} \Delta T(\mathbf{r}, t)$.

Here, $\hbar$ is the reduced Planck constant, $D(\omega)$ is the phonon density of states, $f_{\mathrm{BE}}$ is the Bose-Einstein distribution, and $C_{\omega}=\hbar \omega D(\omega) \frac{\partial f_{\mathrm{BE}}}{\partial T}$ is the mode specific heat. The volumetric heat capacity is then given by $C=\int_{0}^{\omega_{m}} C_{\omega} d \omega$ and the thermal conductivity $k=\int_{0}^{\omega_{m}} k_{\omega} d \omega$, where $k_{\omega}=\frac{1}{3} C_{\omega} v_{\omega} \Lambda_{\omega}$ and $\Lambda_{\omega}=$ $\tau_{\omega} v_{\omega}$ is the phonon MFP.
Both $g_{\omega}$ and $\Delta T$ are unknown. Therefore, to close the problem, energy conservation is used to relate $g_{\omega}$ to $\Delta T$, given by

$$
\iint_{0}^{\omega_{m}}\left[\frac{g_{\omega}(\mathbf{r}, t)}{\tau_{\omega}}-\frac{1}{4 \pi} \frac{C_{\omega}}{\tau_{\omega}} \Delta T(\mathbf{r}, t)\right] d \omega d \Omega=0
$$

where $\Omega$ is the solid angle in spherical coordinates and $\omega_{m}$ is the cutoff frequency. Note that summation over phonon branches is implied without an explicit summation sign whenever an integration over phonon frequency is performed.

Let us now consider solving this equation in an infinite domain or a semi-infinite domain with specular boundary conditions such that the domain can be extended to infinity by symmetry. This assumption eliminates the consideration of diffuse boundaries and finite thickness crystals but still leaves a wide range of problems that can be solved such as multidimensional heat transport in transient grating experiments [29] and the MOSFET devices [19]. In such a domain, a Fourier transform can be applied to all spatial coordinates as well as the time variable, giving

$$
\begin{aligned}
(i \eta & \left.+v_{g} \mu i \xi_{z}+v_{g} \sqrt{1-\mu^{2}} \cos \phi i \xi_{x}+v_{g} \sqrt{1-\mu^{2}} \sin \phi i \xi_{y}\right) \widetilde{g}_{\omega} \\
& =-\frac{\widetilde{g}_{\omega}}{\tau_{\omega}}+\frac{C_{\omega}}{4 \pi \tau_{\omega}} \Delta \widetilde{T}+\frac{\widetilde{Q}_{\omega}}{4 \pi}
\end{aligned}
$$

where a tilde $(\sim)$ over a function indicates the function is in its Fourier-transform form. Here, we define the temporal frequency as $\eta$ and the spatial wave vector in $x, y$, and $z$ as $\xi_{x}$, $\xi_{y}$, and $\xi_{z}$, respectively.

After rearranging Eq. (5), the unknown distribution function is written as

$$
\tilde{g}_{\omega}=\frac{C_{\omega}}{4 \pi} \frac{\Delta \widetilde{T}+\widetilde{Q}_{\omega} \tau_{\omega} / C_{\omega}}{1+i \eta \tau_{\omega}+i \mu \xi_{z} \Lambda_{\omega}+i \sqrt{1-\mu^{2}} \cos \phi \xi_{x} \Lambda_{\omega}+i \sqrt{1-\mu^{2}} \sin \phi \xi_{y} \Lambda_{\omega}} .
$$

Plugging Eq. (6) into Eq. (4), an equation for temperature can be obtained:

$$
\Delta \widetilde{T} \int_{0}^{\omega_{m}} \frac{C_{\omega}}{\tau_{\omega}} d \omega=\frac{1}{4 \pi} \int_{0}^{\omega_{m}} \int_{0}^{2 \pi} \int_{-1}^{1} \frac{C_{\omega}}{\tau_{\omega}} \frac{\Delta \widetilde{T}+\widetilde{Q}_{\omega} \tau_{\omega} / C_{\omega}}{1+i \eta \tau_{\omega}+i \mu \Lambda_{\omega} \xi_{z}+i \sqrt{1-\mu^{2}} \cos \phi \xi_{x} \Lambda_{\omega}+i \sqrt{1-\mu^{2}} \sin \phi \xi_{y} \Lambda_{\omega}} d \mu d \phi d \omega .
$$

The angle integrals on the right-hand side of Eq. (7) can in fact be analytically evaluated using the identities $2.558-4$ and 2.261 in Ref. [30]. Therefore, a simple closed form of temperature in Fourier space can be obtained:

$$
\Delta \widetilde{T}\left(\eta, \xi_{x}, \xi_{y}, \xi_{z}\right)=\frac{\int_{0}^{\omega_{m}} \frac{\widetilde{Q}_{\omega}}{\Lambda_{\omega} \xi} \tan ^{-1}\left(\frac{\Lambda_{\omega} \xi}{1+i \eta \tau_{\omega}}\right) d \omega}{\int_{0}^{\omega_{m}} \frac{C_{\omega}}{\tau_{\omega}}\left[1-\frac{1}{\Lambda_{\omega} \xi} \tan ^{-1}\left(\frac{\Lambda_{\omega} \xi}{1+i \eta \tau_{\omega}}\right)\right] d \omega},
$$

where $\xi=\sqrt{\xi_{x}^{2}+\xi_{y}^{2}+\xi_{z}^{2}}$. Note that if the spectral volumetric heat generation $Q_{\omega}$ is a Dirac-delta function in time and space, Eq. (8) becomes the impulse temperature response or the Green's function of the frequency-dependent BTE. Once $\Delta \widetilde{T}$ is determined, $\widetilde{g}_{\omega}$ can be obtained by Eq. (6). The spectral heat flux is calculated as $\widetilde{\mathbf{q}}_{\omega}=\int \widetilde{g}_{\omega} v_{g} d \Omega$, thus closing the problem. Solutions to each of these quantities in the real-space solution can be easily obtained by inverse Fourier transform.
This solution has exactly the same form as a solution we reported earlier for one spatial dimension except that $\xi$ replaces $\xi_{x}$ in the $1 \mathrm{D}$ solution [31]. At first glance, the fact that an exact analytical solution to the multidimensional BTE exists, and that it is of exactly the same form as the 1D solution, may seem surprising given the complexity of the integrals in Eq. (7). However, on further reflection this result should be expected because the multidimensional solution must be isotropic, as the original BTE was assumed isotropic, and the multidimensional solution must be reduced to the 1D solution in the appropriate limit. The similarity between the multidimensional and $1 \mathrm{D}$ solutions is thus a natural consequence of these two requirements.

For crystals that are not isotropic, Eq. (8) can be easily generalized to include $a b$ initio data using the work of Vermeersch et al. [32], which has been extended to highly anisotropic solids by Minnich [33]. Note that these prior 
works only considered one spatial dimension. By replacing the phonon frequency integral with summation over the phase space, temperature in Fourier space is given by

$$
\Delta \widetilde{T}\left(\eta, \xi_{x}, \xi_{y}, \xi_{z}\right)=\frac{\sum_{\mathbf{k}} \widetilde{Q}_{\mathbf{k}} \gamma_{\mathbf{k}}^{-1}}{\sum_{\mathbf{k}} C_{\mathbf{k}} \tau_{\mathbf{k}}^{-1}\left(1-\gamma_{\mathbf{k}}^{-1}\right)},
$$

where $\mathbf{k}=\left(k_{x}, k_{y}, k_{z}\right)$ is the phonon wave vector, $\gamma_{\mathbf{k}}=1+$ $i \eta \tau_{\mathbf{k}}+i \xi_{x} \Lambda_{x \mathbf{k}}+i \xi_{y} \Lambda_{y \mathbf{k}}+i \xi_{z} \Lambda_{z \mathbf{k}}$, and $\Lambda_{i \mathbf{k}}=v_{i \mathbf{k}} \tau_{\mathbf{k}}$ is the component of the MFP along the $i$ th direction. $C_{\mathbf{k}}$ and $\widetilde{Q}_{\mathbf{k}}$ are the mode specific heat and heat generation, respectively. In this way, full phonon dispersions obtained from $a b$ initio calculations can be directly applied to account for potential crystal anisotropies. This solution is valid for transient transport occurring in all three spatial dimensions.

We next confirm that our solution reduces to the Green's function of the diffusive and ballistic regimes. As previously reported in our earlier $1 \mathrm{D}$ solution, these regimes can be specified by the two nondimensional parameters, the Knudsen number $\mathrm{Kn}_{\omega}=\xi \Lambda_{\omega}$ and transient number $\Gamma_{\omega}=\eta \tau_{\omega}$ in Eq. (8). In the diffusive limit, length and time scales are much larger than the phonon MFPs and relaxation times, respectively, corresponding to $\mathrm{Kn}_{\omega} \ll 1$ and $\Gamma_{\omega} \ll 1$. Examining Eq. (8) under this limit, we find that in Eq. (8)

$$
\frac{1}{\Lambda_{\omega} \xi} \tan ^{-1}\left(\frac{\Lambda_{\omega} \xi}{1+i \eta \tau_{\omega}}\right) \sim 1-i \eta \tau_{\omega}+\frac{1}{3} \Lambda_{\omega}^{2} \xi^{2}
$$

and the solution reduces to the Fourier solution, given by

$$
\Delta \widetilde{T}_{f} \approx \frac{\widetilde{Q}_{0}}{i \eta C+\xi^{2} k},
$$

where $\widetilde{Q}_{0}=\int_{0}^{\omega_{m}} \widetilde{Q}_{\omega} d \omega$. In the ballistic regime, lengths and times are much smaller than MFPs and relaxation times, or $\mathrm{Kn}_{\omega} \gg 1$ and $\Gamma_{\omega} \gg 1$. The BTE thus becomes

$$
\frac{\partial g_{\omega}}{\partial t}+\mathbf{v}_{g} \cdot \nabla g_{\omega}=0
$$

the solution of which is the same as Eq. (8) under the limit of $\tau_{\omega} \rightarrow \infty$, given by

$$
\Delta \widetilde{T}_{b} \approx \frac{1}{\int_{0}^{\omega_{m}} \frac{C_{\omega}}{\tau_{\omega}} d \omega} \int_{0}^{\omega_{m}} \frac{\widetilde{Q}_{\omega}}{\Lambda_{\omega} \xi} \tan ^{-1}\left(\frac{v_{\omega} \xi}{i \eta}\right) d \omega .
$$

Between these two limits is the quasiballistic regime for which we must use the full expression given by Eq. (8).

\section{ADVANTAGES OF THE METHOD}

The BTE has long been a formidable equation due to its high dimensionality and the efficient solution of this equation has been a long-standing challenge in the thermal sciences. Prior solution approaches in the thermal science literature are either extremely simplified or solely numerical and usually computationally expensive. Independent of any numerical considerations, this identification of an analytical solution to BTE that enables physical insight to be obtained just from examining the form of the equation is a useful advance.

Further, the computational effort to obtain a solution for a specific problem is dramatically reduced as compared to that of discrete ordinates which has been typically used in the past. For example, the typical computational time for a problem with a heat impulse in a semi-infinite substrate using discrete ordinates [24] is on the order of $5 \mathrm{~h}$ while our approach takes less than $5 \mathrm{~s}$, a speedup exceeding three orders of magnitude. Furthermore, our result is valid for transient heat conduction in all three dimensions, a situation that has rarely been considered even numerically as the memory requirements for discrete ordinates in multiple dimensions are prohibitively large.

\section{APPLICATIONS}

We now demonstrate the utility of our solution by solving three multidimensional problems for which the only previous solution methods were computationally expensive numerical approaches. We perform our calculations for crystalline silicon using the density functional theory (DFT) dispersion and lifetimes calculated by J. Carrete and N. Mingo using ShengBTE [34,35] and Phonopy [36] from interatomic force constants obtained with VASP [37-40]. The details about converting the $a b$ initio calculations to isotropic dispersion can be found in the Appendix.

\section{A. Transient transport}

The first case we consider here is the transient temperature decay of an infinite silicon crystal subject to a heat impulse with a Gaussian spatial profile. The spectral volume heat generation is given by

$$
Q_{\omega}(x, y, z, t)=\Delta T(0) \frac{C_{\omega}}{\tau_{\omega}} e^{-\pi \frac{x^{2}+y^{2}+z^{2}}{R^{2}}} \delta(t),
$$

where $R$ is the radius of the Gaussian profile as illustrated in the inset of Fig. 1(a). To simplify the analytical expressions, we choose the amplitude of spectral distribution of heat generation to be $\Delta T(0) \frac{C_{\omega}}{\tau \omega}$ corresponding to a thermal distribution. Note that this input quantity can be varied according to the different circumstances.

After transforming Eq. (14) into Fourier space and inserting into Eq. (8), we obtain the temperature profile in Fourier space, given by

$$
\begin{aligned}
& \Delta \widetilde{T}\left(\eta, \xi_{x}, \xi_{y}, \xi_{z}\right) \\
& =\Delta T(0) R^{3} e^{-\frac{R^{2} \xi^{2}}{4 \pi}} \frac{\int_{0}^{\omega_{m}} \widetilde{Q}_{\omega} \frac{1}{\Lambda_{\omega} \xi} \tan ^{-1}\left(\frac{\Lambda_{\omega} \xi}{1+i \eta \tau_{\omega}}\right) d \omega}{\int_{0}^{\omega_{m}} \frac{C_{\omega}}{\tau_{\omega}}\left[1-\frac{1}{\Lambda_{\omega} \xi} \tan ^{-1}\left(\frac{\Lambda_{\omega} \xi}{1+i \eta \tau_{\omega}}\right)\right] d \omega} .
\end{aligned}
$$

Now, we examine how temperature decays with time at the origin. We note that to obtain this quantity it is not necessary to inverse Fourier transform the three spatial wave vectors. Instead, we evaluate the Fourier transform only at $x=y=z=0$ by integrating Eq. (8) over all wave vectors as

$$
\begin{aligned}
\Delta \widetilde{T}(\eta, \mathbf{r}=0)= & \frac{1}{(2 \pi)^{3}} \int_{-\infty}^{\infty} \int_{-\infty}^{\infty} \int_{-\infty}^{\infty} \\
& \times \Delta \widetilde{T}\left(\eta, \xi_{x}, \xi_{y}, \xi_{z}\right) d \xi_{x} d \xi_{y} d \xi_{z} .
\end{aligned}
$$

In this way, only one inverse Fourier transform over the frequency variable is required to obtain the temperature decay.

The resulting transient temperature decay curves are plotted in Fig. 1. In the diffusive limit where the heat generation region is big enough such that $\mathrm{Kn}_{\omega} \ll 1$ and $\Gamma_{\omega} \ll 1$, the BTE solution recovers the Fourier's law solution as in Fig. 1(a). 

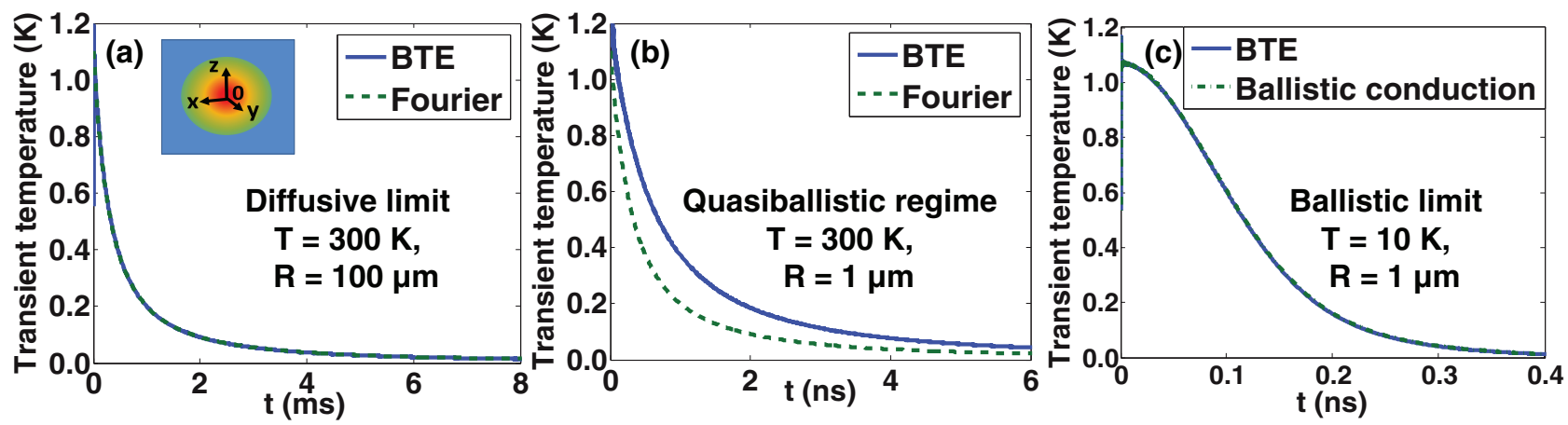

FIG. 1. (Color online) Temperature decay curves $\Delta T(t)$ at the origin for an infinite silicon sample subject to an impulsive volumetric heat generation with Gaussian spatial profile at the origin in the (a) diffusive limit, (b) quasiballistic regime, and (c) ballistic limit. The BTE, Fourier, and ballistic conduction solutions are given by the solid lines, dashed lines, and dash-dotted line, respectively. Inset in (a): 2D schematic of the domain. $R$ is the Gaussian radius.

As the Gaussian radius $R$ decreases and MFPs become comparable or greater than $R$, we observe a deviation of the BTE solution from Fourier's law due to quasiballistic transport, as shown in Fig. 1(b). We note that the actual temperature rise is larger than the Fourier law prediction, consistent with a prior result by Chen [17]. The ballistic limit is plotted in Fig. 1(c), demonstrating that the calculated temperature decay agrees with the ballistic solution of the BTE given by Eq. (13).

\section{B. Quasisteady line heating}

The second case we consider is a line heater on a semiinfinite silicon substrate as illustrated in the inset of Fig. 2(a), where $x$ is the in-plane direction and $z$ is the cross-plane direction. The surface of the sample is subject to a periodic line
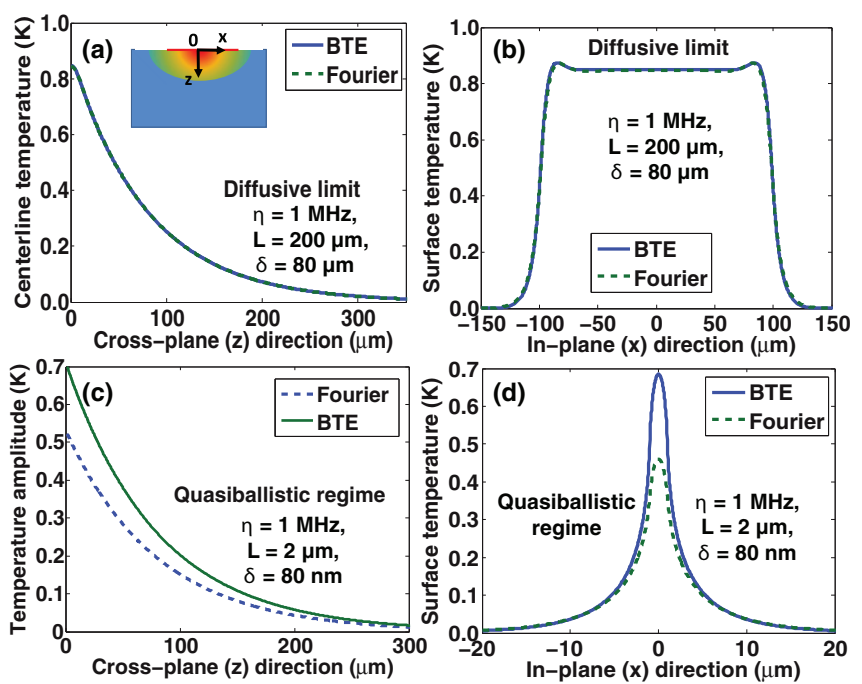

FIG. 2. (Color online) Amplitudes of the temperature decay curves $|\Delta T|$ for a planar semi-infinite silicon sample subject to a periodic line heater at the surface. (a) and (b) are in the diffusive regime while (c) and (d) are in the quasiballistic regime. The BTE and Fourier solutions are given by the solid and dashed lines, respectively. Inset in (a): 2D schematic of the geometry. Phonons incident on the plane at $z=0$ are specularly reflected. The width of line heater is $L$ with an exponential decay with penetration depth $\delta$ in the cross-plane direction, and $\eta$ is the temporal frequency. heating with width $L$ in the $x$ direction, exponential decay with penetration depth $\delta$ in the $z$ direction, and temporal frequency $\eta$. Qualitatively, this geometry corresponds to the geometry for the three- $\omega$ method, a common method used to measure thermal properties [41]. We assume that phonons at $z=0$ are specularly reflected, leading to the adiabatic boundary condition $g_{\omega}(z=0, x, t, \mu)=g_{\omega}(z=0, x, t,-\mu)$. Therefore, by symmetry we can extend the domain to the negative $z$ plane and apply our solution.

The spectral volumetric heat generation in this case is expressed as

$$
\begin{aligned}
Q_{\omega}(x, z, t)= & \Delta T(0) \frac{C_{\omega}}{\tau_{\omega}} e^{i \eta t} e^{-|z| / \delta} \\
& \times\left[H\left(x+\frac{L}{2}\right)-H\left(x-\frac{L}{2}\right)\right],
\end{aligned}
$$

where $H$ is the Heaviside step function. The same procedure as in the first case is carried out to obtain the temperature in Fourier space, which is given by

$$
\begin{aligned}
\Delta \widetilde{T}\left(\eta, \xi_{x}, \xi_{y}, \xi_{z}\right)= & \Delta T(0) \frac{2 \delta}{1+\delta^{2} \xi_{z}^{2}} L \operatorname{sinc}\left(\frac{\xi_{x} L}{2}\right) \\
& \times \frac{\int_{0}^{\omega_{m}} \widetilde{Q}_{\omega} \frac{1}{\Lambda_{\omega} \xi} \tan ^{-1}\left(\frac{\Lambda_{\omega} \xi}{1+i \eta \tau_{\omega}}\right) d \omega}{\int_{0}^{\omega_{m}} \frac{C_{\omega}}{\tau_{\omega}}\left[1-\frac{1}{\Lambda_{\omega} \xi} \tan ^{-1}\left(\frac{\Lambda_{\omega} \xi}{1+i \eta \tau_{\omega}}\right)\right] d \omega} .
\end{aligned}
$$

Since the heating is periodic in time, the temperature profile is quasistationary. We obtain the centerline temperature $[T(x=$ $0, z)$ ] by integrating Eq. (18) over all $\xi_{x}$ and the surface temperature $[T(x, z=0)]$ by integrating Eq. (18) over all $\xi_{z}$.

The centerline temperature amplitudes are shown in Figs. 2(a) and 2(c), and the surface temperature amplitudes are shown in Figs. 2(b) and 2(d). Again, in the diffusive limit, the BTE solutions agree with the Fourier solutions when the heating length is much larger than the phonon MFPs such that $\mathrm{Kn}_{\omega} \ll 1$ and $\Gamma_{\omega} \ll 1$. The phonon transport becomes quasiballistic when the MFPs are comparable to or greater than the characteristic length scales, in which case the BTE solutions no longer agree with the Fourier law solutions. We again observe that the BTE solution predicts a higher temperature rise than Fourier's law in the quasiballistic case 

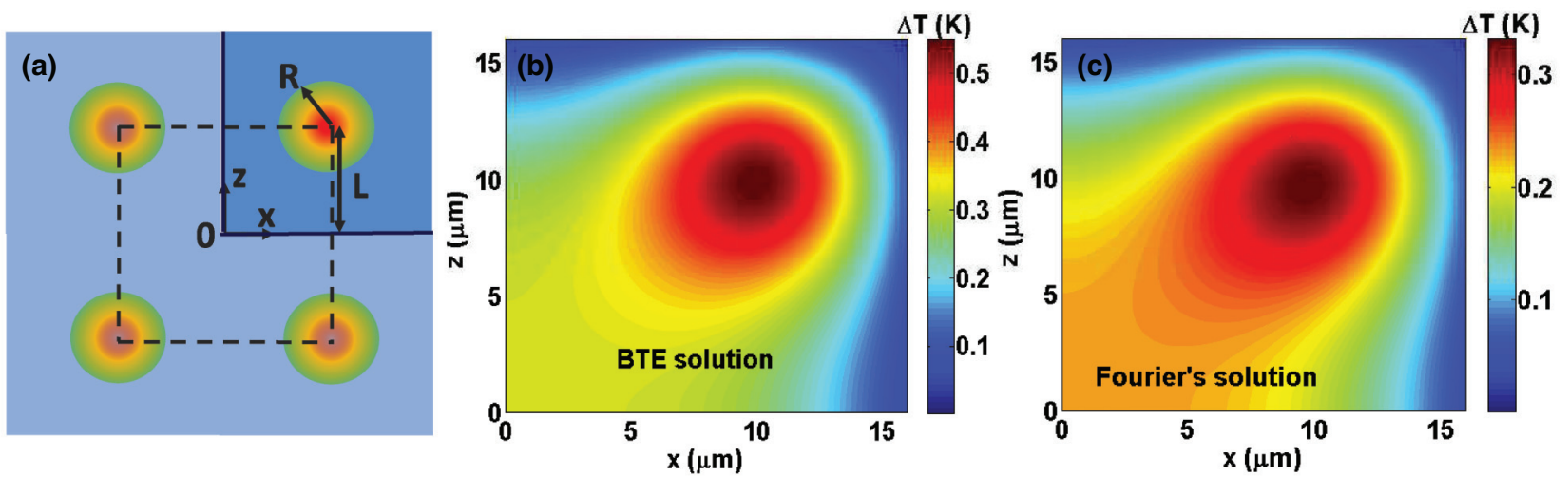

FIG. 3. (Color online) (a) A planar domain located in the first quadrant subject to a Gaussian volumetric heat generation displaced away from the origin. The heating profile is uniform in the cross-plane direction. The radius of the Gaussian profile is $R$ and the distance from the center of the heat to the $x / z$ axis is $L$. The problem is treated using the method of images in which the specular boundaries at $z=0$ and $x=0$ are replaced with three identical heating sources located at the mirror locations across each symmetry plane. The amplitude of $2 \mathrm{D}$ temperature field $|\Delta T(x, z)|$ is given by (b) the BTE and (c) Fourier's law in the quasiballistic regime with $L=10 \mu \mathrm{m}$ and $R=1 \mu \mathrm{m}$.

due to a lack of scattering local to the heater. This observation demonstrates that the three- $\omega$ method must be used with caution when the heater linewidth is comparable to phonon MFPs as interpreting the data with Fourier's law will lead to an incorrect thermal conductivity value.

\section{The method of images}

The last case we consider here is a steady displaced planar Gaussian heating source next to an adiabatic specularly reflecting corner as illustrated in Fig. 3(a). While this geometry is complex, the problem can be effectively solved using the method of images as in elementary electrostatics [42]. To implement the method of images, we replace the specular boundaries with three identical heating sources located at the mirror locations across each symmetry plane. By symmetry and uniqueness of the solution for the linear BTE, this solution is equal to that of the original problem. The method of images is a general technique that can be easily applied to even more complex geometries than that considered here.

The spectral volumetric heat generation for the original and mirror heaters can be expressed as

$$
\begin{aligned}
Q_{\omega}(x, z, t)= & \Delta T(0) \frac{C_{\omega}}{\tau_{\omega}}[f(x, z)+f(-x,-z) \\
& +f(x,-z)+f(-x, z)],
\end{aligned}
$$

where $f(x, z)=e^{-\pi \frac{(x-L)^{2}+(z-L)^{2}}{R^{2}}}, L$ is the displaced distance from the center of the heat to the $z / x$ axis, and $R$ is the radius of the Gaussian profile.

Figures 3(b) and 3(c) give the amplitude of the temperature field when the heater is placed $10 \mu \mathrm{m}$ away from both $x$ and $z$ axes based on the BTE and Fourier's law, respectively. At the given Gaussian radius $(R=1 \mu \mathrm{m})$, the phonon transport is quasiballistic. The peak amplitude of temperature predicted by the BTE is higher than that of Fourier's law. Also, due to a lack of scattering events local to the heater, heat is trapped around the heating source while it diffuses away from the heater by Fourier's prediction. In the past, the only method to solve this problem was purely numerical. Using our solution, we can write the exact solution in Fourier space, and we can obtain the real space solution including frequency-dependent phonon properties in under 1 min on a typical desktop computer.

\section{Other applications}

Besides the three cases we consider above, there are many other geometries in real applications that can be treated as an infinite or semi-infinite domain. One example is multidimensional transient thermal grating experiments [29], which includes a sinusoidal heating profile in the in-plane direction and an exponential heating decay in the cross-plane direction for a semi-infinite solid. Another example is heat generation and transport in the MOSFET devices, which is similar to the second case studied above and has been previously computed using the expensive finite-difference method [19]. Moreover, our Green's-function approach can be also applied to much more complicated geometries as studied in the third case using the method of images, which further extends the scope of the applications of this method.

\section{SUMMARY}

We have presented a new analytical Green's function of the multidimensional frequency-dependent BTE and demonstrated its capability to efficiently solve a wide range of problems that were previously intractable except using expensive numerical approaches. Our solution is valid from the diffusive to ballistic regimes, rigorously includes frequency dependence, and can be applied to a wide range of geometries in three spatial dimensions. Our work will lead to a better understanding of phonon heat conduction over the full range of transport regimes.

\section{ACKNOWLEDGMENTS}

This work was sponsored in part by Robert Bosch, LLC, through Bosch Energy Research Network Grant No. 13.01.CC11, by the National Science Foundation under Grant No. CBET CAREER 1254213, and by Boeing under the Boeing-Caltech Strategic Research and Development Relationship Agreement. 


\section{APPENDIX: ISOTROPIC DISPERSION}

We reduce computational cost by taking advantage of the cubic symmetry of $\mathrm{Si}$ and computing an isotropic equivalent dispersion in phonon frequency $\omega$ space. To obtain this dispersion, we start with the full dispersion in threedimensional wave-vector $k$ space calculated by J. Carrete and N. Mingo using ShengBTE [34,35] and Phonopy [36] from interatomic force constants obtained with VASP [37-40]. Phonon frequencies $\omega_{\mathbf{k}}$, group velocities $\mathbf{v}_{\mathbf{k}}$, and relaxation times $\tau_{\mathbf{k}}$ for the three acoustic and three optical branches are functions of wave vector $\mathbf{k}=\left(k_{x}, k_{y}, k_{z}\right)$. We discretize the phonon frequency between its minimum and maximum values into 101 bins with equal weight $\Delta \omega$. For each polarization, the density of states for a given frequency bin $\omega_{i}$ is obtained by counting the number of modes $N$ that fall into that bin, given by

$$
D\left(\omega_{i}\right)=\frac{N}{V \Delta \omega},
$$

where $V$ is the volume of the first Brillouin zone, calculated from the maximum wave vectors given by the data.

We obtain average group velocities using $v_{\text {avg }}^{2}\left(\omega_{i}\right)=$ $\left\langle\mathbf{v}^{2}\left(\omega_{i}\right)\right\rangle$, or the average of the square of group velocity for phonons in a specific frequency bin. In addition, we can compute the average of the square of the speed in a particular direction, i.e., $v_{x}^{2}\left(\omega_{i}\right)=\left\langle v_{k_{x}}^{2}\left(\omega_{i}\right)\right\rangle$. We find that $v_{x}^{2}\left(\omega_{i}\right)=v_{y}^{2}\left(\omega_{i}\right)=v_{z}^{2}\left(\omega_{i}\right)=\frac{1}{3} v_{\mathrm{avg}}^{2}\left(\omega_{i}\right)$, which confirms that the dispersion of $\mathrm{Si}$ is isotropic on average.

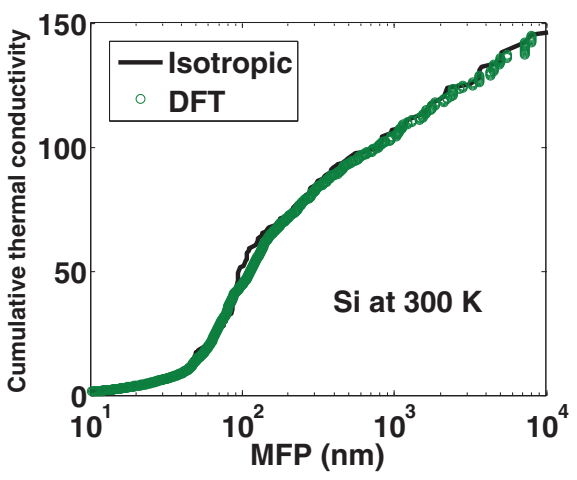

FIG. 4. (Color online) Cumulative thermal conductivity as a function of phonon mean free path (MFP) for silicon at $300 \mathrm{~K}$ calculated by the isotropic equivalent dispersion (solid line) and the original DFT data (circles). The isotropic dispersion matches well with the ab initio calculations as expected for a cubic crystal.

To obtain the average relaxation time, we compute $\left\langle\tau\left(\omega_{i}\right) \mathbf{v}^{2}\left(\omega_{i}\right)\right\rangle / v_{\text {avg }}^{2}\left(\omega_{i}\right)$. This particular averaging is chosen to maintain the spectral thermal conductivity distribution of the full dispersion.

The final bulk thermal conductivity for this isotropic equivalent dispersion of silicon at $300 \mathrm{~K}$ is $167.0 \mathrm{~W} / \mathrm{m} \mathrm{K}$, very close to the original value of $166.7 \mathrm{~W} / \mathrm{m} \mathrm{K}$, and the cumulative thermal conductivity spectra are nearly identical, as shown in Fig. 4.
[1] D. G. Cahill, P. V. Braun, G. Chen, D. R. Clarke, S. Fan, K. E. Goodson, P. Keblinski, W. P. King, G. D. Mahan, A. Majumdar, H. J. Maris, S. R. Phillpot, E. Pop, and L. Shi, Appl. Phys. Rev. 1, 011305 (2014).

[2] K. Biswas, J. He, I. D. Blum, C.-I. Wu, T. P. Hogan, D. N. Seidman, V. P. Dravid, and M. G. Kanatzidis, Nature (London) 489, 414 (2012).

[3] R. J. Mehta, Y. Zhang, C. Karthik, B. Singh, R. W. Siegel, T. Borca-Tasciuc, and G. Ramanath, Nat. Mater. 11, 233 (2012).

[4] E. Pop, Nano Res. 3, 147 (2010).

[5] A. Majumdar, J. Heat Transfer 115, 7 (1993).

[6] G. Chen, Nanoscale Energy Transport and Conversion (Oxford University, New York, 2005).

[7] S. Chandrasekhar, Radiative Transfer (Dover, New York, 1950).

[8] K. M. Case, Ann. Phys. 9, 1 (1960).

[9] C. E. Siewert and P. F. Zweifel, Ann. Phys. 36, 61 (1966).

[10] M. M. R. Williams, Nucl. Sci. Eng. 18, 260 (1964).

[11] R. Peierls, Ann. Phys. 3, 1055 (1929).

[12] R. Englman, Proc. Phys. Soc. 72, 391 (1958).

[13] M. M. R. Williams, J. Phys. D 4, 1201 (1971).

[14] S. Simons, J. Phys. C 4, 2089 (1971).

[15] G. D. Mahan and Francisco Claro, Phys. Rev. B 38, 1963 (1988).

[16] A. A. Joshi and A. Majumdar, J. Appl. Phys. 74, 31 (1993).

[17] G. Chen, J. Heat Transfer 118, 539 (1996).

[18] G. Chen, Phys. Rev. Lett. 86, 2297 (2001).
[19] R. Yang, G. Chen, M. Laroche, and Y. Taur, J. Heat Transfer 127, 298 (2005).

[20] J. Ordonez-Miranda, R. Yang, and J. J. Alvarado-Gil, J. Appl. Phys. 109, 084319 (2011).

[21] K. Esfarjani, G. Chen, and H. T. Stokes, Phys. Rev. B 84, 085204 (2011).

[22] D. A. Broido, A. Ward, and N. Mingo, Phys. Rev. B 72, 014308 (2005).

[23] S. V. J. Narumanchi, J. Y. Murthy, and C. H. Amon, J. Heat Transfer 125, 896 (2003).

[24] A. J. Minnich, G. Chen, S. Mansoor, and B. S. Yilbas, Phys. Rev. B 84, 235207 (2011).

[25] Q. Hao, G. Chen, and M.-S. Jeng, J. Appl. Phys. 106, 114321 (2009).

[26] L. Chaput, Phys. Rev. Lett. 110, 265506 (2013).

[27] J.-P. M. Peraud and N. G. Hadjiconstantinou, Phys. Rev. B 84, 205331 (2011).

[28] J.-P. M. Peraud and N. G. Hadjiconstantinou, Appl. Phys. Lett. 101, 153114 (2012).

[29] J. A. Johnson, A. A. Maznev, M. T. Bulsara, E. A. Fitzgerald, T. C. Harman, S. Calawa, C. J. Vineis, G. Turner, and K. A. Nelson, J. Appl. Phys. 111, 023503 (2012).

[30] I. S. Gradshteyn and I. M. Ryzhik, Table of Integrals, Series and Products (Academic, New York, 1980).

[31] C. Hua and A. J. Minnich, Phys. Rev. B 89, 094302 (2014). 
[32] B. Vermeersch, J. Carrete, N. Mingo, and A. Shakouri, arXiv:1406.7341.

[33] A. J. Minnich, arXiv:1409.5364.

[34] W. Li, N. Mingo, L. Lindsay, D. A. Broido, D. A. Stewart, and N. A. Katcho, Phys. Rev. B 85, 195436 (2012).

[35] W. Li, J. Carrete, N. A. Katcho, and N. Mingo, Comput. Phys. Commun. 185, 1747 (2014).

[36] A. Togo, Phonony, v1.8.5, http://phonopy.sourceforge.net
[37] G. Kresse and J. Hafner, Phys. Rev. B 48, 13115 (1993).

[38] G. Kresse and J. Hafner, Phys. Rev. B 49, 14251 (1994).

[39] G. Kresse and J. Furthmuller, Comput. Mater. Sci. 6, 15 (1996).

[40] G. Kresse and J. Furthmüller, Phys. Rev. B 54, 11169 (1996).

[41] D. G. Cahill, Rev. Sci. Instrum. 61, 802 (1990).

[42] D. J. Griffiths, Introduction to Electrodynamics, 3rd ed. (Prentice-Hall, Upper Saddle River, NJ, 1999). 